\title{
Acupuncture as an intervention to reduce alcohol dependency: a systematic review and meta-analysis
}

\author{
Charlotte Southern' ${ }^{1}$, Charlie Lloyd ${ }^{1}$, Jianping Liu², Congcong Wang ${ }^{2}$, Tingting Zhang ${ }^{1}$, Martin Bland ${ }^{1}$
} and Hugh MacPherson ${ }^{1 *}$ (D)

\begin{abstract}
Background: Acupuncture has been widely used as a treatment for alcohol dependence. An updated and rigorously conducted systematic review is needed to establish the extent and quality of the evidence on the effectiveness of acupuncture as an intervention for reducing alcohol dependence. This review aimed to ascertain the effectiveness of acupuncture for reducing alcohol dependence as assessed by changes in either craving or withdrawal symptoms.

Methods: In this systematic review, a search strategy was designed to identify randomised controlled trials (RCTs) published in either the English or Chinese literature, with a priori eligibility criteria. The following English language databases were searched from inception until June 2015: AMED, Cochrane Library, EMBASE, MEDLINE, PsycINFO, and PubMed; and the following Chinese language databases were similarly searched: CNKI, Sino-med, VIP, and WanFang. Methodological quality of identified RCTs was assessed using the Jadad Scale and the Cochrane Risk of Bias tool.

Results: Fifteen RCTs were included in this review, comprising 1378 participants. The majority of the RCTs were rated as having poor methodological rigour. A statistically significant effect was found in the two primary analyses: acupuncture reduced alcohol craving compared with all controls (SMD $=-1.24,95 \% \mathrm{Cl}=-1.96$ to -0.51$)$; and acupuncture reduced alcohol withdrawal symptoms compared with all controls (SMD $=-0.50,95 \% \mathrm{Cl}=-0.83$ to $-0.17)$. In secondary analyses: acupuncture reduced craving compared with sham acupuncture (SMD $=-1.00,95 \%$ $\mathrm{Cl}=-1.79$ to -0.21 ); acupuncture reduced craving compared with controls in RCTs conducted in Western countries $(\mathrm{SMD}=-1.15,95 \% \mathrm{Cl}=-2.12$ to -0.18$)$; and acupuncture reduced craving compared with controls in RCTs with only male participants (SMD $=-1.68,95 \% \mathrm{Cl}=-2.62$ to -0.75$)$.

Conclusion: This study showed that acupuncture was potentially effective in reducing alcohol craving and withdrawal symptoms and could be considered as an additional treatment choice and/or referral option within national healthcare systems.
\end{abstract}

\section{Background}

Approximately 3.3 million deaths worldwide are attributed to alcohol dependency per year [1]. The prevalence of alcohol dependency in the UK in 2010 was estimated at $5.9 \%$ of the population (8.7\% of men and $3.2 \%$ of women), compared with $4 \%$ in Europe $[1,2]$. A dependent drinker who stops drinking will experience alcohol withdrawal

\footnotetext{
*Correspondence: hugh.macpherson@york.ac.uk

1 Department of Health Sciences, University of York, York, UK

Full list of author information is available at the end of the article
}

syndrome; this is a distressing and life-threatening condition with symptoms that range in severity, including tremors, agitation, paroxysmal sweats, fever, nausea, and seizures [3]. These symptoms typically occur within the first $24 \mathrm{~h}$ of stopping drinking and can last a number of weeks [4].

In England, treatment for alcohol dependency is received by a minority (6\%) of an estimated 1 million people aged 16-65 years who are alcohol dependent [2]. The UK National Institute for Health and Care Excellence (NICE) reports that non-pharmacological treatments are 
an important therapeutic option for people with alcoholrelated problems, and that acupuncture is valued by service users with alcohol-related problems [2]; however, NICE acknowledges that the evidence base for the effectiveness of acupuncture is weak [2].

A preliminary search of the field identified two reviews specifically related to acupuncture as an intervention for alcohol disorders [5, 6]. The review by Kunz et al. [5] included 14 studies investigating the effectiveness of auricular acupuncture in the treatment of withdrawal from substances (opiate, cocaine, and alcohol). The authors decided not to conduct a meta-analysis owing to potential systematic and selection biases. The findings for the review were inconclusive and the effectiveness of auricular acupuncture as an intervention for withdrawal was not determined. The included studies lacked rigorous methodology, resulting in reduced internal validity. In this review, Chinese language studies were excluded.

A subsequent review by Cho and Whang [6] included 11 studies and did not limit acupuncture techniques to auricular acupuncture [6]. A meta-analysis for treatment completion rates identified no statistically significant difference between acupuncture and either sham or no acupuncture groups. The results were equivocal and the included studies lacked rigorous methodology. However, the review included languages other than English, which increased its robustness.

An updated review is needed, using rigorous review methods. In the present review, we refined the search for acupuncture as a treatment for alcohol craving and withdrawal symptoms in alcohol-dependent individuals. We included randomised controlled studies (RCTs) published in both the English and Chinese literature, and conduct meta-analyses on the main outcome measures. Therefore, the present review expands on the existing research in this area to provide fresh and relevant evidence from all RCTs to establish whether acupuncture is effective in reducing alcohol craving and withdrawal symptoms.

\section{Methods}

Eligibility of studies for this systematic review

Inclusion and exclusion criteria were pre-specified (Table 1).

\section{Search method}

The following English language databases were searched up to June 2015: AMED (OvidSP) (from 1985), the Cochrane Library, EMBASE (OVID) (from 1946), MEDLINE (OVID) (from 1946), PsycINFO (from 1987), and PubMed (from 1970); and the following Chinese language database were searched up to June 2015: CNKI (from 1994), Sino-med (from 1960), VIP (from 1989), and WanFang (from 1998). Table 2 presents the keywords used, and Appendix S1 (Additional file 1) provides the MeSH terms and keywords used in the Medline (OVID) search.

\section{Study selection}

CS screened the Western databases; titles and abstracts were analysed to exclude irrelevant and duplicate studies. CW screened the Chinese literature using the same criteria. All relevant studies were retrieved as full reports for detailed evaluation. Any study that did not satisfy the inclusion criteria was excluded.

\section{Data extraction}

Data were extracted by CS on study design, participant characteristics, results, and statistical information. Translations of the full Chinese reports were undertaken by TZ at the University of York; these translations were clarified by CS, with further clarification from CW.

\section{Quality assessment}

The included RCTs were assessed for bias and scored using the Cochrane Risk of Bias tool [7], and for methodological quality using the Jadad Scale [8].

\section{Narrative synthesis}

A narrative synthesis was performed to provide a basis for assessing each RCTs contribution to the research question in terms of interpretation, synthesis, and triangulation regarding the quantitative data.

\section{Quantitative synthesis}

Meta-analyses produced the overall effect estimates for the two primary outcomes related to craving and withdrawal symptoms. Continuous outcomes (Visual Analogue Scale and Likert-scaled options used to assess craving and Alcohol Withdrawal Syndrome Scale, the

Table 1 Defined inclusion and exclusion criteria

\begin{tabular}{ll}
\hline Population & Alcohol dependents, inpatients and outpatients. Animal studies excluded \\
Intervention & Acupuncture where the needle punctures the skin surface at acupuncture points; either auricular or body \\
Control & Sham acupuncture or treatment as usual or other treatment \\
Outcomes & Primary measures: alcohol craving and alcohol-withdrawal symptoms \\
Study design & Secondary: adverse effects \\
\hline
\end{tabular}


Table 2 Key terms (or nearest appropriate Chinese equivalent)

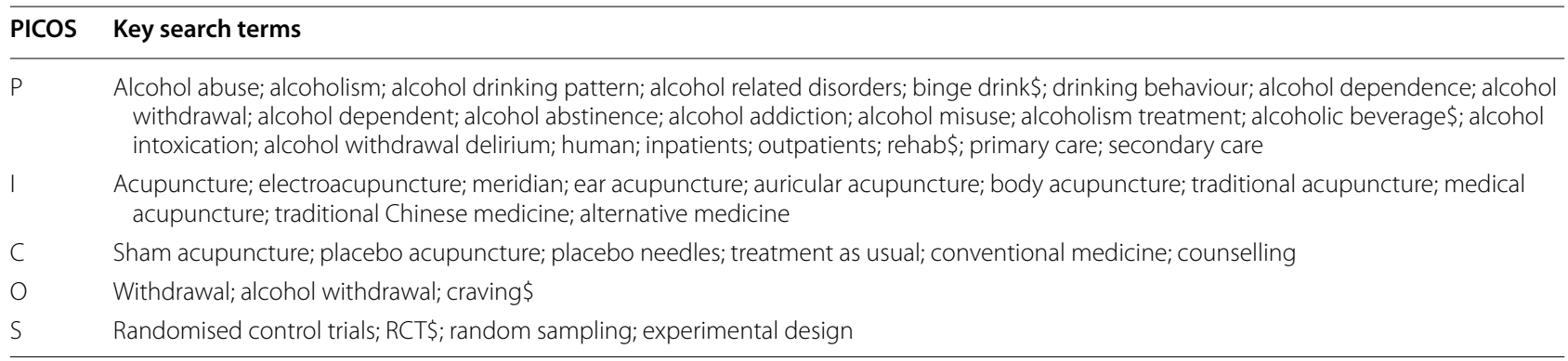

Mainz Alcohol Withdrawal Scale, and the Clinical Institute Withdrawal Assessment for withdrawal) were presented as the standardised mean difference (SMD) with 95\% confidence intervals (CI). As each RCT did not use the same acupuncture technique, we could not assume that acupuncture in each RCT was estimating the same effect, therefore we used a random-effects model for meta-analyses. For RCTs containing either two acupuncture groups or two sham acupuncture groups, both arms were combined for the analyses. Analyses of data on craving and withdrawal outcomes at the end of the intervention phase were performed using Comprehensive Meta-Analysis Version 2 software (Biostat Inc, Englewood, NJ, USA). Forest plots were drawn using our own routines in Stata (Stata Corp, College Station, TX, USA)

\section{Assessment of heterogeneity}

Clinical heterogeneity was explored through the narrative synthesis, identifying any variations in participants and interventions. Clinical heterogeneity was present in each meta-analysis owing to differences in the acupuncture techniques used and participant status (inpatient or outpatient) in the experimental interventions. Statistical heterogeneity was investigated for $\mathrm{I}^{2}$ statistics above $50 \%$, i.e. demonstrative of moderate heterogeneity [9]. Secondary analyses based on variations in RCT characteristics were used to explore potential sources of heterogeneity.

\section{Results}

\section{Description of included studies}

Overview

This updated review included 15 RCTs with a total of 1378 participants; Table 3 lists the characteristics of included RCTs. Figure 1 details the PRISMA flowchart of this review.

\section{Design}

All of the studies were RCTs with a parallel study design. Most RCTs involved two arms, with the exception of three RCTs that were three-armed [10-12], and one that was four-armed [13].

\section{Participants}

Participants in 12 of the 15 RCTs satisfied one of three definitions of alcohol dependence: the Diagnostic and Statistical Manual of Mental Disorders (DSM)-III-R, the DSM-IV, the International Classification of Diseases (ICD-10), or the Chinese Classification of Mental Disorders (CCMD-3). However, three RCTs used additional selection criteria: one required participants to be inpatients of at least 14 days [13], one required participants to have been drinking within 10 days of enrolment in the outpatient alcoholism treatment programme [12], and one did not specify a definition, only stated that participants were alcohol-dependent [14]. The participants were inpatients in 11 of 15 RCTs, the exceptions were four RCTs involving outpatients [10, 12, 15, 16], and one RCT in which the setting was not specified [17]. The included RCTs were conducted in the USA $(n=3)$, Europe $(n=6)$, or East Asia $(n=6)$.

\section{Description of acupuncture}

The acupuncture techniques varied between RCTs. Eight RCTs used auricular acupuncture at points recommended by the UK National Acupuncture Detoxification Association (NADA); three of these eight RCTs also used body acupuncture. Seven RCTs used body acupuncture as the active treatment, either conventional needle acupuncture or electroacupuncture. All acupuncture techniques involved skin penetration.

The majority of RCTs (12 of 15) reported that the duration of needling was 30-45 min; two RCTs reported that the duration of needling was $<30 \min [15,18]$, and one RCT did not report the duration of needling [14].

The duration of the treatment courses ranged from 5 days [19] to 12 weeks [17], or until a participant reached the withdrawal stage [11]. Six RCTs had fewer than 10 treatment sessions [10,14, 18-21] two RCTs had 12-18 treatment sessions $[13,15]$, three RCTs had 24-30 


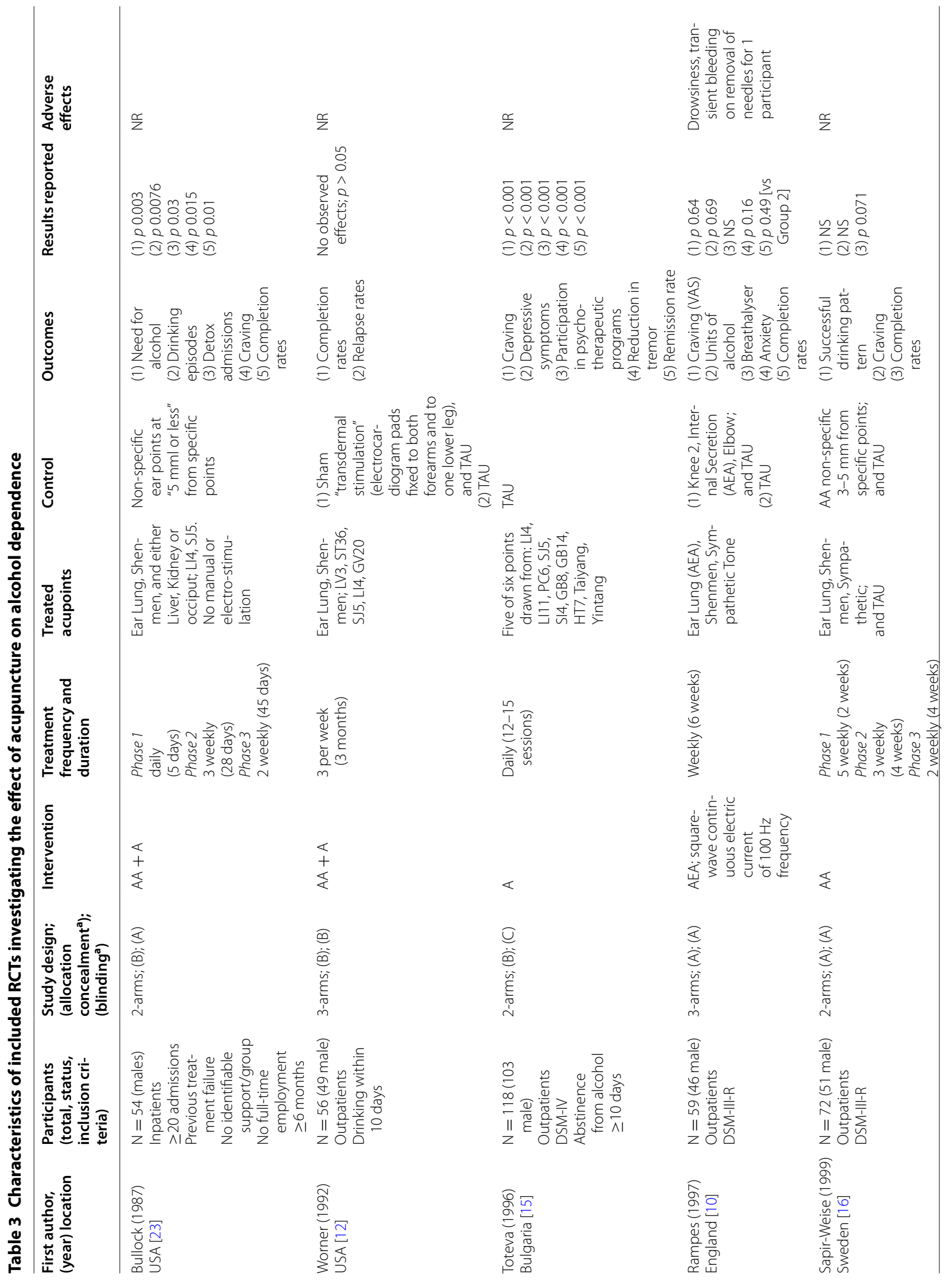




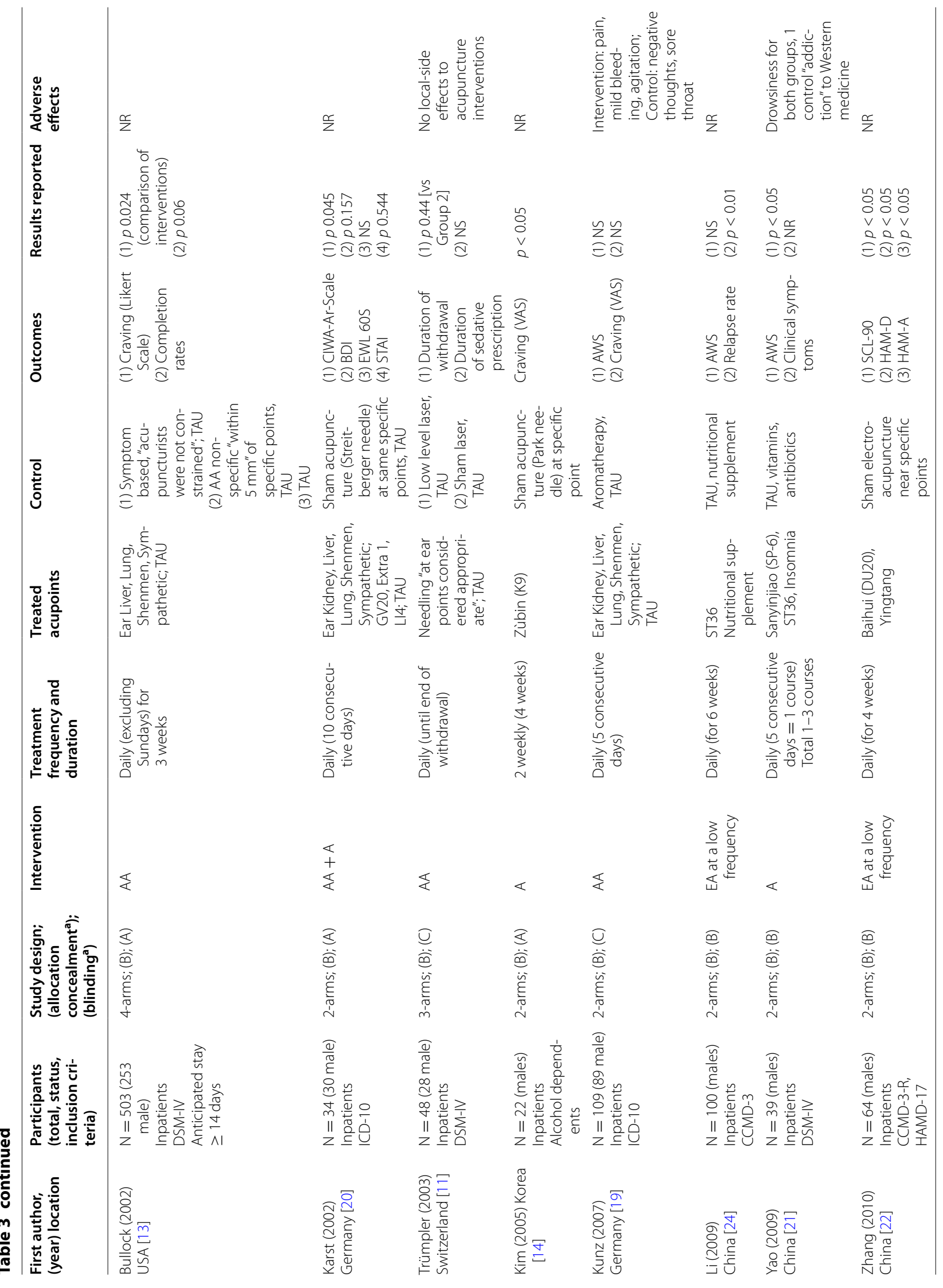




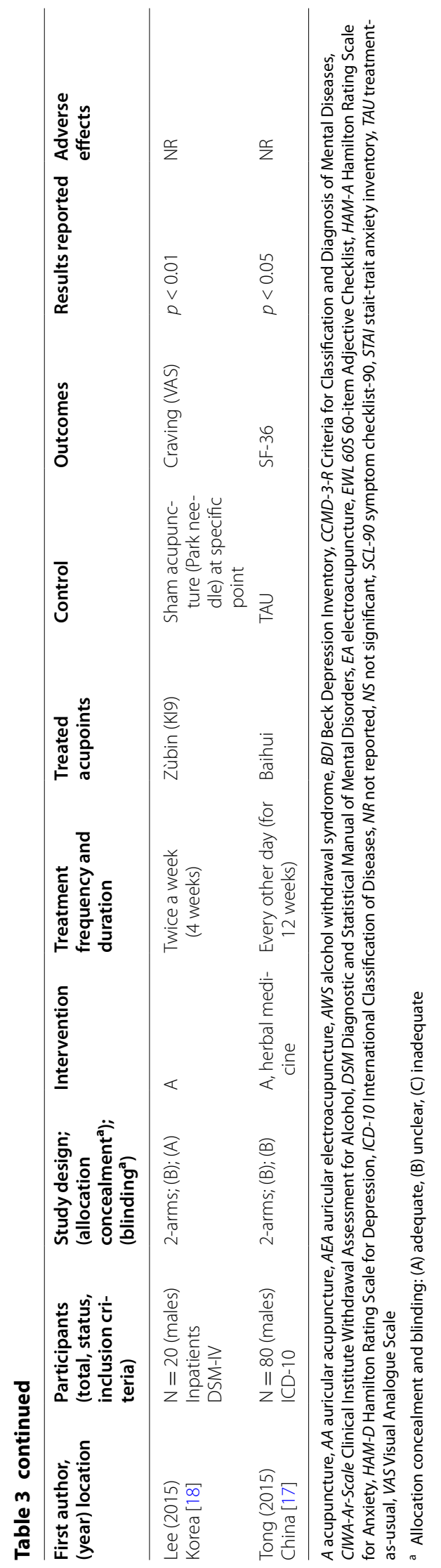




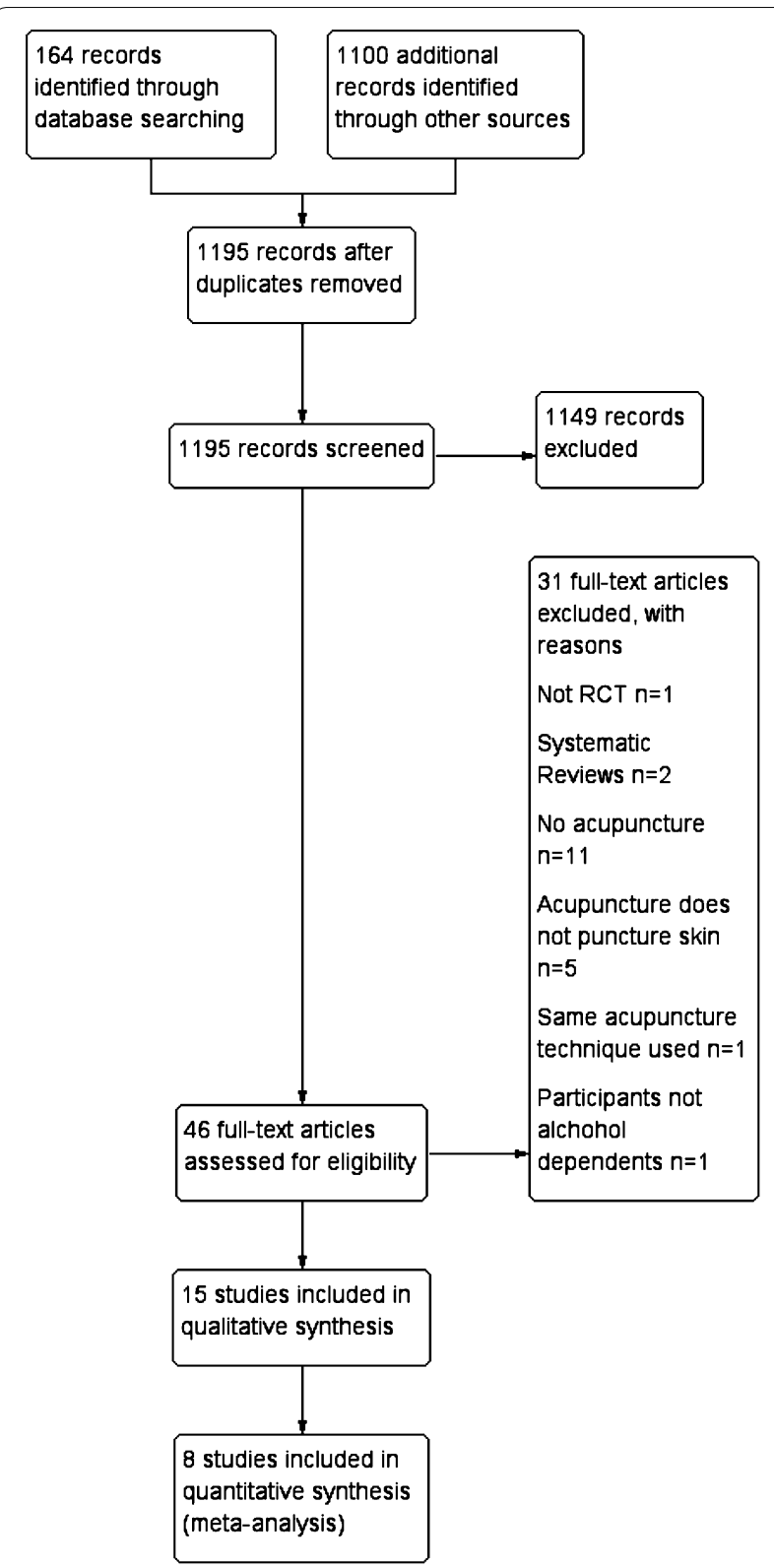

Fig. 1 PRISMA flow diagram detailing the number of studies included and/or excluded at each stage

treatment sessions [16, 22, 23], and three RCTs had 36-42 treatment sessions [12, 17, 24]. One RCT included a varying number of treatment sessions, as the sessions were continued until participants had become abstinent from alcohol [11].

Acupuncture was performed by an acupuncturist or Chinese medical practitioner $(\mathrm{n}=7)$, a registered nursetrained acupuncturist $(\mathrm{n}=1)$, and NADA-trained mental health nurses $(n=1)$; six RCTs did not report these details. Five RCTs reported that interactions between the acupuncturist and participants were constrained by the study design; the majority (10 of $15 \mathrm{RCTs}$ ) did not report this information.

\section{Description of controls}

Ten RCTs used sham acupuncture as a control, with the aim of minimising differences in the treatment experience between groups, with participants blinded to the intervention. Fourteen RCTs used treatment-as-usual as an intervention, either as a control group $(n=5)$ or as an adjunct to the experimental treatment $(\mathrm{n}=9)$. In total there were 20 control groups.

\section{Outcomes}

Alcohol craving and alcohol withdrawal symptoms were measured at key time points in the RCTs: at baseline, during intervention, and at follow-up. Visual Analogue Scale and Likert-scaled options were used to assess craving. The Alcohol Withdrawal Syndrome Scale, the Mainz Alcohol Withdrawal Scale, and the Clinical Institute Withdrawal Assessment were used to assess the severity of withdrawal symptoms. Where data on adverse events were reported, they were collected through observation by acupuncturists or self-reported by participants.

\section{Cochrane Risk of Bias}

Assessments using the Cochrane Risk of Bias tool [7] are presented in Figs. 2 and 3. Details by RCT are provided in Appendix S2 (Additional file 1).

\section{Jadad Scale}

The Jadad Scale [8] scores are presented in Table 4. Five RCTs had a high methodological quality score $(\geq 3)$ and ten RCTs had a low methodological quality score $(\leq 2)$. Owing to the difficulties in blinding the acupuncturist, none of the RCTs were able to score the maximum of five points; therefore a score of three, which is classified as high methodological quality, was the maximum score possible.

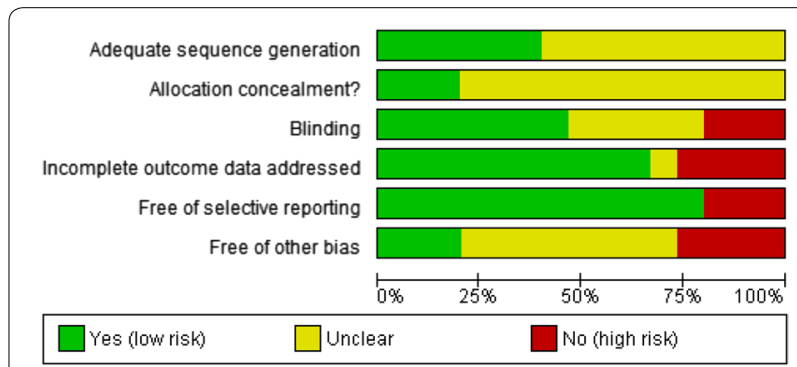

Fig. 2 Risk of Bias: review authors'judgements about each methodological quality item presented as percentage across all studies 


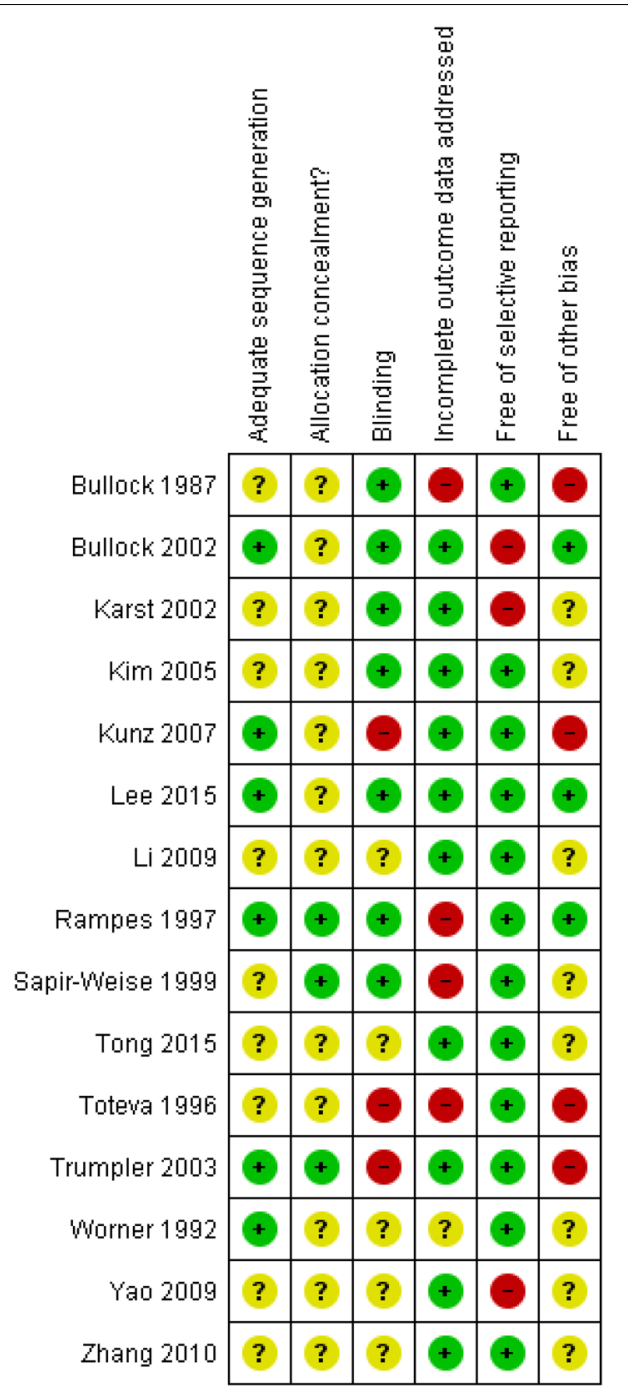

Fig. 3 Risk of bias: review authors'judgements for each domain

\section{Primary meta-analyses}

Craving Nine RCTs assessed alcohol craving. One RCT compared acupuncture with an active treatment (aromatherapy) and was therefore excluded from the analysis as it was considered an equivalent intervention [19]. Only six RCTs [10, 14-16, 18, 23] ( $\mathrm{n}=345$ participants) provided sufficient statistical information to include in the meta-analysis. Five of these RCTs measured craving using a quantitative scale; however, only one RCT provided the odds ratio [23]. Therefore, the odds ratio was converted into the standardised difference, using a method suggested by Borenstein et al. [25], so that the statistical information could be included in the analysis. There was evidence of a statistically significant effect of acupuncture on selfreported experience of craving compared to all control groups (sham and treatment-as-usual) (SMD $=-1.24$,
95\% CI $=-1.96$ to $-0.51, \mathrm{Q}=30.89, \mathrm{df}=5, P<0.001$, $\mathrm{I}^{2}=83.8 \%$; Fig. 4).

Alcohol withdrawal Five RCTs assessed alcohol withdrawal symptoms. Again, the one RCT that compared acupuncture with an active treatment (aromatherapy) was excluded from the meta-analysis [19]. Two further RCTs were excluded as they provided insufficient statistical data $[20,21]$. From the remaining two RCTs [11, 24] ( $n=148$ participants), there was evidence of a significant effect of acupuncture compared to all controls for alcohol withdrawal symptoms (SMD $=-0.50,95 \% \mathrm{CI}=-0.83$ to $-0.17, \mathrm{Q}=0.11, \mathrm{df}=1, P=0.003, \mathrm{I}^{2}=0 \%$; Fig. 5).

\section{Secondary subgroup and sensitivity meta-analyses}

For alcohol craving, the data were split to conduct analyses that might lead to an explanation of the heterogeneity.

Acupuncture versus sham acupuncture for alcohol craving Pooling only those RCTs that compared acupuncture with sham acupuncture ( $\mathrm{n}=211$ participants), acupuncture significantly reduced self-reported experience of craving (SMD $=-1.00,95 \% \mathrm{CI}=-1.79$ to -0.21 , $\mathrm{Q}=17.74, \mathrm{df}=4, \mathrm{P}=0.013, \mathrm{I}^{2}=77.4 \%$; Fig. 6). There was a small reduction in the $\mathrm{I}^{2}$ statistic.

Studies conducted in Western countries for alcohol craving Pooling the endpoint values for the Western-based RCTs ( $n=303$ participants), there was a statistically significant effect of acupuncture on self-reported experience of craving (SMD $=-1.15,95 \% \mathrm{CI}=-2.12$ to -0.18 , $\mathrm{Q}=27.44, \mathrm{df}=3, P=0.02)$, with slightly increased heterogeneity $\left(\mathrm{I}^{2}=89.07 \%\right.$; Fig. 7$)$.

Male-only randomised controlled trials for alcohol craving Out of the $15 \mathrm{RCTs}$ ( $\mathrm{n}=1378$ participants), eight RCTs included females ( $\mathrm{n}=327$ participants) and seven RCTs were male-only studies. An analysis was conducted on three of these male-only RCTs. Pooling the endpoint values, there was a statistically significant effect of acupuncture versus controls on self-reported experience of craving ( $\mathrm{SMD}=-1.68,95 \% \mathrm{CI}=-2.62$ to -0.75 , $\mathrm{Q}=4.46, \mathrm{df}=2, \mathrm{P}<0.001, \mathrm{I}^{2}=55.12 \%$; Fig. 8). Excluding the RCTs containing both male and female participants considerably reduced the heterogeneity.

\section{Adverse events}

Four RCTs reported adverse effects, although data were limited. Kunz et al. [19] reported pain, mild bleeding on insertion of needles, and agitation in the acupuncture group, while the aromatherapy group experienced agitation, sneezing, negative thoughts, and sore throat. Yao [21] reported that drowsiness was experienced in both 
Table 4 Jadad Scale representing scores in descending order

\begin{tabular}{|c|c|c|c|c|c|c|}
\hline Study & $\begin{array}{l}\text { Described } \\
\text { as RCT? }\end{array}$ & $\begin{array}{l}\text { Adequate } \\
\text { randomisation? }\end{array}$ & Double-blind? & $\begin{array}{l}\text { Details of } \\
\text { double-blinding? }\end{array}$ & $\begin{array}{l}\text { Reasons stated } \\
\text { for withdrawals? }\end{array}$ & Total score \\
\hline Bullock et al. [13] & +1 & +1 & +0 & +0 & +1 & 3 \\
\hline Kunz et al. [19] & +1 & +1 & +0 & +0 & +1 & 3 \\
\hline Lee et al. [18] & +1 & +1 & +0 & +0 & +1 & 3 \\
\hline Rampes et al. [10] & +1 & +1 & +0 & +0 & +1 & 3 \\
\hline Trümpler et al. [11] & +1 & +1 & +0 & +0 & +1 & 3 \\
\hline Worner et al. [12] & +1 & +1 & +0 & +0 & +0 & 2 \\
\hline Karst et al. [20] & +1 & -1 & +0 & +0 & +1 & 1 \\
\hline Kim et al. [14] & +1 & -1 & +0 & +0 & +1 & 1 \\
\hline Li and Guo [24] & +1 & -1 & +0 & +0 & +1 & 1 \\
\hline Yao [21] & +1 & -1 & +0 & +0 & +1 & 1 \\
\hline Zhang et al. [22] & +1 & -1 & +0 & +0 & +1 & 1 \\
\hline Bullock et al. [23] & +1 & -1 & +0 & +0 & +0 & 0 \\
\hline Sapir-Weise et al. [16] & +1 & -1 & +0 & +0 & +0 & 0 \\
\hline Toteva and Milanov [15] & +1 & -1 & +0 & +0 & +0 & 0 \\
\hline Tong et al. [17] & +1 & -1 & +0 & +0 & +0 & 0 \\
\hline
\end{tabular}

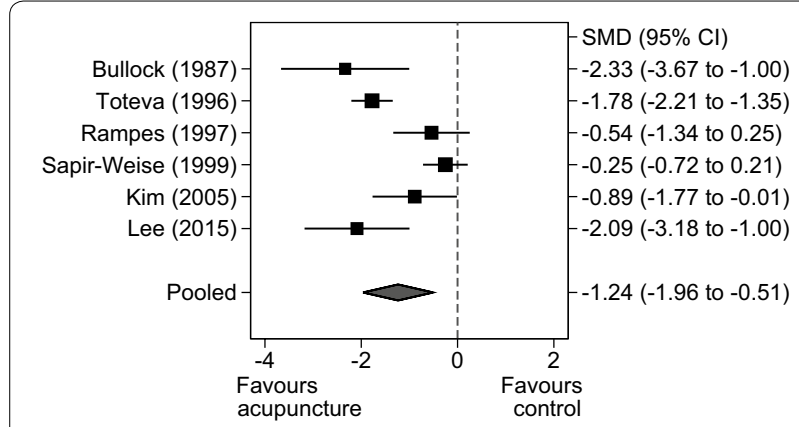

Fig. 4 Forest plot: acupuncture versus all controls (combined sham and treatment as usual) for alcohol craving

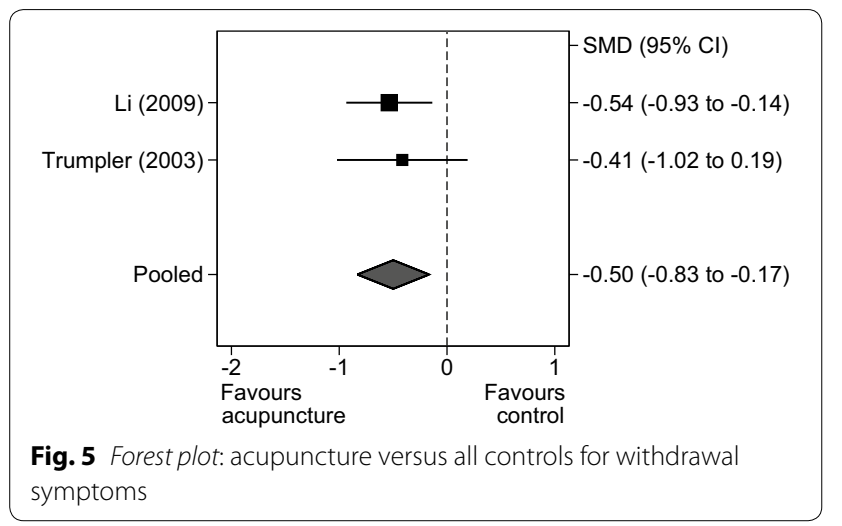

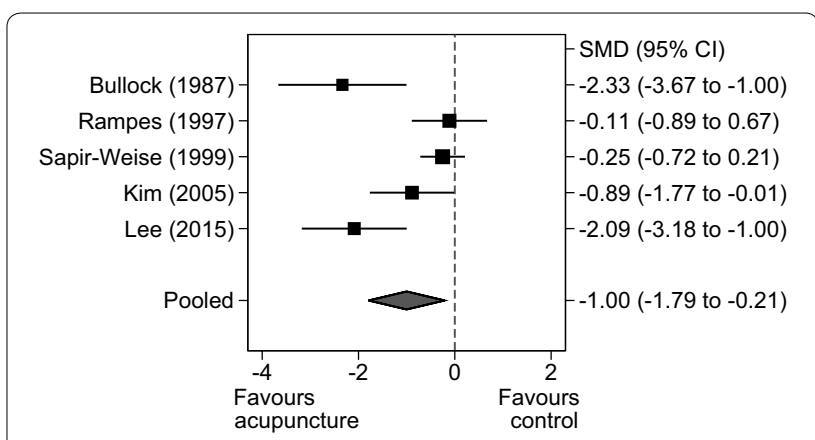

Fig. 6 Forest plot: acupuncture versus sham acupuncture for craving

the acupuncture group $(\mathrm{n}=1)$ and the control group $(\mathrm{n}=4)$; however, one participant in the control group also reported an addiction to pharmaceutical medicine. Rampes et al. [10] reported drowsiness and transient bleeding on removal of needles in the electroacupuncture group at specific acupuncture points; one participant in the non-specific electroacupuncture group dropped out of the RCT owing to pain. Trümpler et al. [11] reported that no local side effects were observed. However, one participant in the acupuncture group experienced self-limiting generalised convulsions whilst sleeping on the fifth day; the convulsions lasted $5 \mathrm{~min}$, and were judged to be a withdrawal-related epileptic seizure on clinical grounds. 


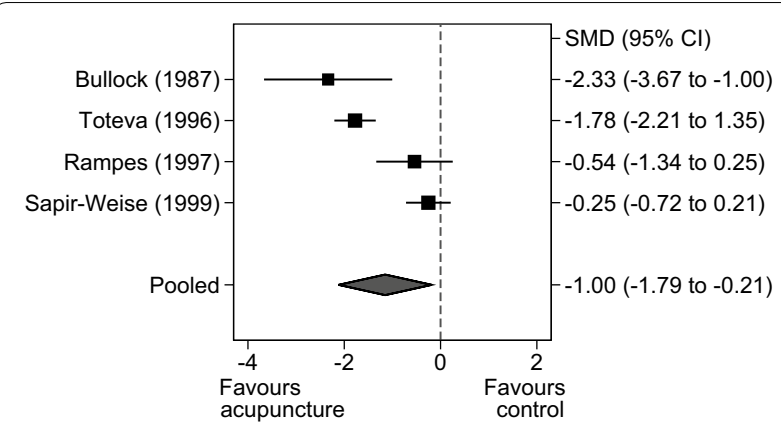

Fig. 7 Forest plot: acupuncture versus control groups for craving in Western studies

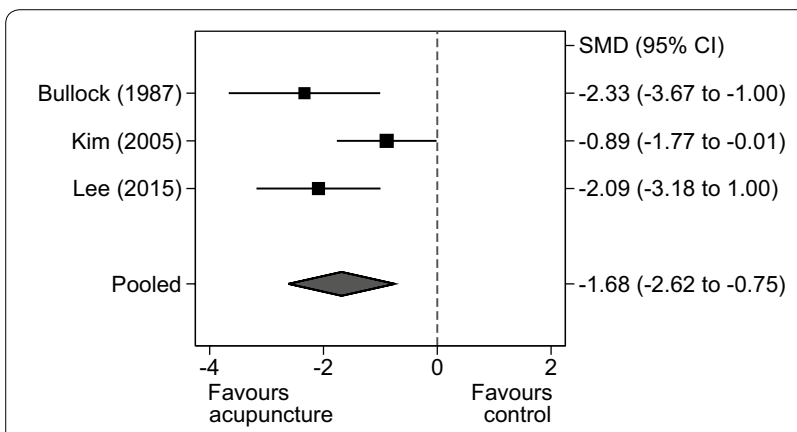

Fig. 8 Forest plot graph: acupuncture versus control groups for craving in male participants

\section{Discussion}

\section{Major findings}

Our review comprised 15 RCTs with 1378 patients from eight countries published in either Chinese or English. Our findings provide the first clear evidence that acupuncture might be considered an effective intervention for alcohol dependence. This evidence of significant effectiveness was observed in our two primary analyses, one showing a reduction in alcohol craving and one showing a reduction in withdrawal symptoms. In all three of our secondary analyses, we found significant reductions in alcohol craving: comparing acupuncture versus sham acupuncture; comparing acupuncture with controls in only those RCTs conducted in Western countries; and in those RCTs that recruited males only. We note that the effect size of acupuncture when all RCTs are combined is slightly larger than when compared with sham acupuncture RCTs or with Western-based RCTs, and is slightly smaller when compared with male-only RCTs. While the overall effect estimate favoured acupuncture for all analyses, some of the individual RCTs found non-significant effects of acupuncture on alcohol dependence. By pooling the data across RCTs, we increased the overall power, which led to the conclusion that acupuncture was an effective intervention.
The present review has identified pre-specified secondary analyses to explore reasons for potential heterogeneity and aid interpretation. The $\mathrm{I}^{2}$ value for acupuncture versus all controls for alcohol craving was $83.8 \%$; this value reduced slightly for the analysis of acupuncture versus sham acupuncture in alcohol craving (77.4\%). An analysis of alcohol craving in Western-based RCTs showed increased heterogeneity (89.1\%). An analysis of acupuncture for male-only participants for alcohol craving substantially reduced the heterogeneity (55.1\%). Interpreting these values with reference to the $50 \%$ threshold [9] indicates that the results must be treated with caution.

\section{Strengths and limitations}

The included RCTs were rigorously assessed using two quality assessments. The included RCTs were not all of a high standard, with 10 of the 15 RCTs scoring two or less on the Jadad scale. Moreover, only eight RCTs contained sufficient statistical information on outcomes for meta-analysis; this limited the scope for comparing acupuncture with some controls. For example, because of a lack of sufficient quantitative outcome data we were unable compare acupuncture with treatment-as-usual. While we have combined changes in continuous outcomes using standardised mean differences for the purposes of conducting the meta-analyses, the individual measures of craving and withdrawal symptoms may have different measurement characteristics, which would lead to an increase in heterogeneity. Furthermore, most of the included RCTs contained a small sample size. These factors resulted in a small number of participants being included in the final analyses. The present review did not include a sufficient number of RCTs to address publication bias.

The majority of the included RCTs were conducted in a hospital setting; therefore, generalisation of the results to community settings must be done with caution. The clinical heterogeneity associated with the different acupuncture techniques limits our ability to identify which technique was more effective for treatment of alcohol disorders. Acupuncture treatments also varied in duration, frequency, and the acupuncture points used, making it difficult to assess the key characteristics that might be associated with the effectiveness of the intervention. Whilst some RCTs combined auricular acupuncture and body acupuncture as part of the same treatment, most RCTs involved either auricular or body acupuncture. Given the potential variation in modes of action, combining these two modalities (either within RCTs or between RCTs), will add to the clinical heterogeneity. Sham acupuncture also varied across RCTs. Moreover, sham acupuncture cannot be considered a physiologically inert 
intervention, thereby potentially leading to an underestimate of the effect of the acupuncture.

\section{Comparisons with previous systematic reviews}

The present review included an increased number of RCTs $(\mathrm{n}=15)$ compared with the previous reviews of Kunz et al. [5] $(n=6)$ and Cho and Whang [6] $(n=11)$. Kunz et al. [5] did not conduct a meta-analysis, and reported that there was insufficient evidence to conclude whether auricular acupuncture is an effective intervention in the treatment of alcohol and substance abuse. Cho and Whang conducted a meta-analysis of acupuncture versus sham acupuncture for treatment completion rates, reporting no significant differences [6] but no metaanalysis was conducted by them for craving because of insufficient data. By contrast, our study found sufficient data to conduct a meta-analysis of craving with six trials included [10, 14-16, 18, 23]. Moreover, the present review is the first to provide a meta-analysis of withdrawal symptoms, albeit based on only two RCTs [11, 24]. The results we present here therefore represent an update of the evidence, with the caveat that our results are based on a small number of RCTs with variable risks of bias.

\section{Implications for research and practice}

Non-pharmacological treatments are an important therapeutic option for people with alcohol-related problems, and there is some evidence that acupuncture is valued by service users [2]. Recommendations for future studies to improve the quality and statistical power of the next review on this topic include:

- Larger sample sizes Further RCTs with larger sample sizes are needed. An increased sample size increases power statistically, enhancing a meta-analysis. Large sample sizes also provide the opportunity to explore potential moderators and mediators of response.

- Relevant statistical information reported Summary statistics on outcomes should be reported to a high standard so that these data can be included, thereby enhancing the robustness of the meta-analyses. An assessment for publication bias could also be conducted using a funnel plot.

- Female participants More studies should recruit female as well as male participants, so that results can be better generalised to the female population of problem drinkers.

- Longer-term follow ups for at least 1 year are recommended, along with parallel cost-effectiveness analyses.

- Increase methodological quality Future studies should follow STRICTA recommendations [26], which are guidelines specifically aimed at producing high quality reporting of acupuncture interventions, and the Consolidated Standards of Reporting Trials (CONSORT) statement [27], which are guidelines on study design and reporting to reduce potential bias and increase methodological quality.

\section{Conclusion}

This study showed that acupuncture was potentially effective in reducing alcohol craving and withdrawal symptoms and could be considered as an additional treatment choice and/or referral option within national healthcare systems.

\section{Additional file}

Additional file 1. Search strategy and risk of bias tables.

\section{Abbreviations}

A: acupuncture; AA: auricular acupuncture; AEA: auricular electroacupuncture; AMED: allied and complementary medicine database; CCMD: Chinese Classification of Mental Disorders; Cl: confidence interval; CNKI: China National Knowledge Infrastructure; CONSORT: consolidated standards of reporting trials; df: degrees of freedom; DSM: Diagnostic and Statistical Manual of Mental Disorders; EA: electroacupuncture; EMBASE: excerpta medica database; ICD: International Classification of Diseases; ${ }^{2}$ : heterogeneity statistic; MEDLINE: national library of medicine database; MeSH: medical subject headings; NADA: national acupuncture detoxification association; NICE: national institute for health and care excellence; NR: not reported; NS: not significant; OVID: OVID database search interface; PsycINFO: psychology information database; PRISMA: preferred reporting items for systematic reviews and meta-analyses; PubMed: search engine for national library of medicine databases; Q: heterogeneity statistic; RCT: randomised controlled trial; Sino-med: Chinese biomedical literature service system; SMD: standardised mean difference; STRICTA: revised standards for reporting interventions in clinical trials of acupuncture; TAU: treatment-as-usual; VIP: Chinese science and technology periodicals database; WanFang: China medical collections database; UK: United Kingdom.

\section{Authors' contributions}

$\mathrm{CS}, \mathrm{CL}$ and $\mathrm{HM}$ conceived the review. CCW, JPL, and CS searched, and with TTZ, extracted the data. MB performed the statistical analysis. CS and HM wrote the manuscript. All authors read and approved the final manuscript.

\section{Author details}

${ }^{1}$ Department of Health Sciences, University of York, York, UK. ${ }^{2}$ Centre for Evidence-Based Chinese Medicine, Beijing University of Chinese Medicine, Beijing, China.

\section{Acknowledgements}

Acknowledgement is due to Janet Eldred for copy-editing the near final version of the manuscript.

\section{Competing interests}

The authors declare that they have no competing interests.

\section{Availability of data and materials}

All data used in this systematic review are fully available in the public domain.

\section{Funding}

The research presented in this study was not funded. Therefore no funding body influenced the design of the study, or the collection, analysis, and interpretation of data on which the manuscript is based.

Received: 10 May 2016 Accepted: 17 November 2016

Published online: 15 December 2016 


\section{References}

1. World Health Organisation. WHO: Global status report on alcohol and health [Internet]. 2014. http://www.who.int/substance_abuse/publications/global_alcohol_report/en/. Accessed 24 Feb 2016.

2. National Institute for Health and Care Excellence (NICE). Alcohol-use disorders: diagnosis, assessment and management of harmful drinking and alcohol dependence | Introduction | Guidance and guidelines [Internet]. 2011. https://www.nice.org.uk/guidance/cg115/chapter/introduction. Accessed 24 Feb 2016.

3. Health and Social Care Information Centre (HSCIC). National Statistics: Statistics on Alcohol - England, 2014 [Internet]. 2014. http://www.hscic. gov.uk/catalogue/PUB14184. Accessed 24 Feb 2016.

4. Rassool GH. Alcohol and drug misuse: a handbook for students and health professionals. 1st ed. Oxford: Routledge; 2009.

5. Kunz S, Schulz M, Syrbe G, Driessen M. Acupuncture of the ear as therapeutic approach in the treatment of alcohol and substance abuse-a systematic review. Sucht. 2004;50:196-203.

6. Cho S-H, Whang W-W. Acupuncture for alcohol dependence: a systematic review. Alcohol Clin Exp Res. 2009;33:1305-13.

7. Higgins JPT, Altman DG, Gøtzsche PC, Jüni P, Moher D, Oxman AD, et al. The Cochrane collaboration's tool for assessing risk of bias in randomised trials. BMJ. 2011;343:d5928.

8. Jadad AR, Moore RA, Carroll D, Jenkinson C, Reynolds DJ, Gavaghan DJ, et al. Assessing the quality of reports of randomized clinical trials: is blinding necessary? Control Clin Trials. 1996;17:1-12.

9. Higgins JP, Thompson SG. Quantifying heterogeneity in a meta-analysis. Stat Med. 2002;21:1539-58.

10. Rampes H, Pereira S, Mortimer A, Manoharan S, Knowles M. Does electroacupuncture reduce craving for alcohol? A randomized controlled study. Complement Ther Med. 1997;5:19-26.

11. Trümpler F, Oez $S$, Stähli $P$, Brenner HD, Jüni P. Acupuncture for alcohol withdrawal: a randomized controlled trial. Alcohol Alcohol. 2003:38:369-75

12. Worner TM, Zeller B, Schwarz H, Zwas F, Lyon D. Acupuncture fails to improve treatment outcome in alcoholics. Drug Alcohol Depend. 1992;30:169-73.

13. Bullock ML, Kiresuk TJ, Sherman RE, Lenz SK, Culliton PD, Boucher TA, et al A large randomized placebo controlled study of auricular acupuncture for alcohol dependence. J Subst Abuse Treat. 2002;22:71-7.

14. Kim S, Kang C, Park J, Kim M, Jung T, Park J, et al. The effect of acupuncture on Zùbin (K9) in reducing alcohol craving in alcohol-dependent patients. Eur Neuropsychopharmacol. 2005;15:S580.
15. Toteva S, Milanov I. The use of body acupuncture for treatment of alcohol dependence and withdrawal syndrome: a controlled study. Am J Acupunct. 1996;24:19-26.

16. Sapir-Weise R, Berglund M, Frank A, Kristenson H. Acupuncture in alcoholism treatment: a randomized out-patient study. Alcohol Alcohol. 1999;34:629-35.

17. Tong $X$, Zhao F, Tong Z. Observation on Chinese herbal medicine with acupuncture therapies for alcohol dependence syndrome. JCAM 2015:31:24-5.

18. Lee J-S, Kim S-G, Jung T-G, Jung WY, Kim S-Y. Effect of Zhubin (KI9) acupuncture in reducing alcohol craving in patients with alcohol dependence: a randomized placebo-controlled trial. Chin J Integr Med. 2015;21:307-11.

19. Kunz S, Schulz M, Lewitzky M, Driessen M, Rau H. Ear acupuncture for alcohol withdrawal in comparison with aromatherapy: a randomizedcontrolled trial. Alcohol Clin Exp Res. 2007;31:436-42.

20. Karst M, Passie T, Friedrich S, Wiese B, Schneider U. Acupuncture in the treatment of alcohol withdrawal symptoms: a randomized, placebocontrolled inpatient study. Addict Biol. 2002;7:415-9.

21. Yao P. Integrated traditional and western medicine treatment for alcoholic cirrhosis: compensatory period combined with alcohol dependence syndrome in 23 cases. Chin J Integr Tradit West Med Liver Disease. 2009;19:364-5.

22. Zhang $Y$, Zhang $R$, Chen $Y$, Wang $H$, Tan Q. Observation on therapeutic effect of electroacupuncture for treatment of emotional disturbance of patients with alcohol dependence syndrome in hospital. Med J Chin People's Health. 2010;22:1197-8.

23. Bullock ML, Umen AJ, Culliton PD, Olander RT. Acupuncture treatment of alcoholic recidivism: a pilot study. Alcohol Clin Exp Res. 1987;11:292-5.

24. Li G, Guo J. Studies of low frequency electroacupuncture treat for patients with alcohol dependence. World Health Digest Med Periodical. 2009;8:27-8.

25. Borenstein M, Hedges L, Higgins J, Rothstein H. Introduction to metaanalysis. Chichester: Wiley; 2009

26. MacPherson $H$, Altman DG, Hammerschlag $R$, Youping $L$, Taixiang W, White A, et al. Revised standards for reporting interventions in clinical trials of acupuncture (STRICTA): extending the CONSORT statement. PLoS Med. 2010;7:e1000261.

27. Schulz KF, Altman DG, Moher D, for the CONSORT Group. CONSORT 2010 statement: updated guidelines for reporting parallel group randomised trials. BMJ. 2010;340:c332.

\section{Submit your next manuscript to BioMed Central and we will help you at every step:}

- We accept pre-submission inquiries

- Our selector tool helps you to find the most relevant journal

- We provide round the clock customer support

- Convenient online submission

- Thorough peer review

- Inclusion in PubMed and all major indexing services

- Maximum visibility for your research

Submit your manuscript at www.biomedcentral.com/submit
BioMed Central 